\title{
CFD-Based process optimization of a dissolved air flotation system for drinking water production
}

\author{
K. Satpathy, U. Rehman, B. Cools, L. Verdickt, G. Peleman \\ and I. Nopens wa
}

\begin{abstract}
Dissolved air flotation (DAF) has received more attention recently as a separation technique in both drinking water as well as wastewater treatment. However, the process as well as the preceding flocculation step is complex and not completely understood. Given the multiphase nature of the process, fluid dynamics studies are important to understand and optimize the DAF system in terms of operation and design. The present study is intended towards a comprehensive computational analysis for design optimization of the treatment plant in Kluizen, Belgium. Setting up the modeling framework involving the multiphase flow problem is briefly discussed. 3D numerical simulations on a scaled down model of the DAF design were analysed. The flow features give better confidence, but the flocs escape through the outlet still prevails which is averse to the system performance. In order to improve the performance and ease of maintenance, design modifications have been proposed by using a perforated tube for water extraction and are found to be satisfactory. The discussion is further reinforced through validating the numerical model against the experimental findings for stratified flow conditions.

Key words | acoustic doppler velocimetry, CFD, DAF, flocs, multiphase flow

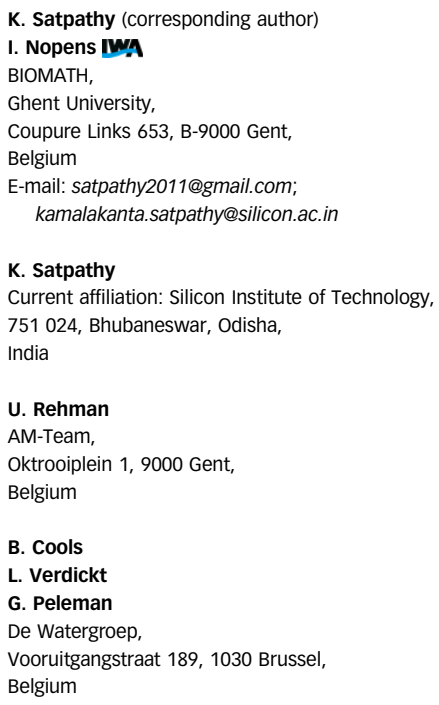

\section{INTRODUCTION}

Dissolved air flotation (DAF) is an effective process in drinking water and wastewater treatment and has been used in many spheres of industry (Edzwald I995). The process is the opposite of sedimentation. Both methods rely on density differences to remove solids; however, in sedimentation, solids sink to the bottom whereas in a DAF tank, they are transported upward to the surface of the tank to form a dense sludge layer. The latter is achieved by injecting air bubbles via air injectors under high pressure into the main solids laden water stream. The bubbles attach to the flocs and reduce their density, making them rise to the reactor surface. The sludge layer that develops at the surface is thereafter removed by scrapers or by overflow and the clear water is discharged at the outlet.

The design and operation of DAF plants is largely based on experience and pilot plant data. Understanding of DAF has been greatly improved through development of fundamental principles and numerical models. Some key contributions in this respect are those of Edzwald et al. (I990), Edzwald (I995). Haarhoff \& Edzwald (2004) have summarized the state of knowledge regarding modelling doi: $10.2166 /$ wst.2020.028 the contact and separation zones of the DAF process and have identified areas that need additional research. DAF plants until 1990 were designed with long pretreatment flocculation times and with low hydraulic loadings $(\sim 5-10 \mathrm{~m} / \mathrm{h})$. In the last 15 years, there has been a trend of reducing the flocculation retention time and increasing the hydraulic loading rate. At present, the need for information about the DAF process has gone beyond these guidelines and further research is of crucial importance. A stratified flow pattern has been recently identified as a favourable mechanism for the flotation process. However, not all DAF units run optimally and they can still suffer from recirculation, causing a disruption of the flow, resulting in a decreased efficiency.

A general consensus within the water industry is that there is a need for research towards optimization of the DAF process and reliable design criteria, which would increase the level of safe quality and decrease the costs for investment and operation. Following the evolution of the computational fluid dynamics (CFD) codes in the last decade, several investigators have employed numerical 
simulations to examine various aspects of flotation processes (Guichao et al. 2018). Owing to the substantial computational burden, most of them refer to two-phase (gas-liquid) systems and only a few attempts have been made with three coexisting phases (gas-liquid-solid). Fawcett (1997) studied the two-phase hydraulics of a DAF tank in a 2D frame of reference using the CFX 4 code. Among the examined parameters (tank dimensions, baffle arrangements, water and air flow rates and the turbulence governing the collision and adhesion of the particles to air bubbles), it was found that the injected air-to-water flow momentum ratio was the most critical design parameter dictating the effective mixing of air with the main liquid stream. To achieve a stratified flow, the velocity from the needle valves was reduced. The latter choice may be debatable, as Fawcett (1997) mentioned that the most important parameter for the DAF process is the transfer of momentum between air and water. Another recent 2D model, presented by Emmanouil et al. (2007), has demonstrated a malfunctioning DAF tank producing macrobubbles. However, the authors did not compare their results with experimental data. As pointed out by Amato \& Wicks (2007), small or no changes at all seen in the CFD model might turn out to give major effects when tested experimentally. The fact that a geometrical design of a tank can be optimized using the CFD approach was previously shown by Kwon et al. (2006) who found that an optimal length to width ratio is important for the efficiency of the process. In their work, a standard $k-\varepsilon$ turbulence model was used, but the resolution of the flow field in the vicinity of the walls was not addressed in detail. Note that there is a possibility that the selection of the turbulence model is less significant when making a fullscale model in which the wall effects will not be as dominant as in a small scale model. Bondelind et al. (2010) discussed the modelling framework and identified a number of parameters relevant when setting up a CFD simulation of a DAF tank.

With respect to experimental data collection, only a limited number of studies have been performed given the complex nature of the multi-phases involved. Few attempts were made to analyse the flow features, especially the velocity distribution in the DAF, using the acoustic doppler velocimetry (ADV), laser doppler velocimetry (LDV) and the particle image velocimetry (PIV) techniques. But, all the experimental observations were investigated, mainly in the two phase scenarios. Ta \& Brignal (1997) successfully proved the result of the two-phase (gas and liquid) CFD interpretation using ADV. However, the experimental verification of the velocity with the CFD results were not so accurate due to turbulence. Lundh (2000) measured the local water velocities in a DAF pilot plant, and presented the results from measurements done in the separation zone using an ADV. A test of the ADV showed that the bubble content and the hydraulic loading were significantly influencing the flow pattern. Hague et al. (200I) performed a simulation for small-scale DAF where the length of a gun is less than $1 \mathrm{~m}$ and verified the result using the LDV and PIV. Kwon et al. (2006) investigated the effect of length/width (L/W) on the hydrodynamic behaviour using CFD and ADV technique in a full scale, two-phase DAF system. Based on the results, they concluded that the L/W ratio and the outlet geometry play an important role for flow pattern and fine bubble distribution in the flotation zone.

From the above-cited literature, it is observed that very few attempts were made on the 3D numerical multiphase aspect of DAF systems. The present study is intended as a necessary step towards a comprehensive computational model for design and optimation of the Kluizen DAF plant as well as providing guidance for future DAF designs within De Watergroep. In summary, the work is carried out as follows: first, a brief description of the present water treatment plant is given. Next, the set-up of the modelling phenomena in a 3D numerical domain is discussed. In the results and discussion section, flow features in the DAF systems are analysed and validated against the experimental findings. Finally, design modifications for the future treatment plants are systematically tested and evaluated.

\section{NUMERICAL MODEL AND SOLUTION METHODOLOGY}

\section{Status of the present DAF plant, Kluizen}

The treatment plant at Kluizen, Belgium is currently operated at $20 \mathrm{ML} /$ day to produce drinking water from reservoir water, characterised by high levels of organic matter and alkalinity. The design value for the maximum nominal hydraulic loading is $13.6 \mathrm{~m} / \mathrm{h}$. However, due to high effluent turbidity, it is difficult to operate the plant at its design capacity. To improve the effluent turbidity at high loading, the flotation units were equipped with lamella separators in the separation zones. The raw water is coagulated using $30 \mathrm{mg} / \mathrm{L}$ of sulphuric acid and $11 \mathrm{mg} / \mathrm{L}$ of PACl. Flocculation takes place in a series of three flocculators, equipped with paddle wheel stirrers. Subsequently, the water enters the flotation units. Saturated water is produced in a packed bed saturator and injected into the contact zone 
Table 1 | Design parameters and operational settings of the Kluizen DAF plant

\begin{tabular}{ll}
\hline $\begin{array}{l}\text { Flow rate }\left(\mathrm{m}^{3} / \mathrm{h}\right) \\
\text { Coagulation and flocculation (two parallel units) }\end{array}$ & $400-1,000$ \\
Sulfuric acid dose $(\mathrm{mg} / \mathrm{L})$ & 30 \\
PACl dose $(\mathrm{mg} \mathrm{Al} / \mathrm{L})$ & 11 \\
Total flocculation volume $\left(\mathrm{m}^{3}\right)$ & 240 \\
Minimum flocculation time (min) & 28 \\
Flotation (two parallel units) & \\
Length, width of the contact zone $(\mathrm{m})$ & $1.44,5.13$ \\
Length, width of the separation zone $(\mathrm{m})$ & $5.80,5.13$ \\
Water height in the contact zone $(\mathrm{m})$ & 2.80 \\
Water height in the separation zone $(\mathrm{m})$ & $1.80-2.05$ \\
Number of flotation nozzles in the contact zone & 40 \\
Saturation flow $(\%)$ & 6 \\
\hline
\end{tabular}

of the flotation unit by means of 40 pneumatically adjustable nozzles (Verdickt et al. 2016). After flotation, filtration takes place in four dual media filters. After dual media filtration, the water is treated further by means of ozonation, granular activated carbon filtration and $\mathrm{NaOCl}$ disinfection. The design parameters and the operational settings of the treatment plant are summarized in Table 1.

\section{NUMERICAL MODEL}

\section{Steady transient simulation}

It is of great importance to clearly define the conditions for which a steady/transient analysis is appropriate. For example, the development of the flow field just after the bubbles have been injected is clearly time dependent. On the other hand, once the general flow pattern is established, it is reasonable to assume that the spatial distribution of the continuous flow properties is invariant with time. Furthermore, in a DAF tank, if we consider the turbulent kinetic energy for illustration, it can be argued that the change of kinetic energy will be considerable, for example, in regions close to the injection point of the recycle flow. However, the overall turbulent kinetic energy in the tank is considered relatively low, since no external forces, e.g. in the form of stirring, are added to the system. Such conditions justify a steady state analysis in the flotation system. This will increase the speed of the simulations and enable much faster convergence, especially for the 3D cases.

\section{D 3D Numerical analysis}

Another important question for the analysis to be performed is the choice between performing 2D or 3D simulations. Literature reviews within the field of numerical simulations of DAF show a clear overrepresentation of the former. It is obvious that the exclusion of the third dimension decreases the computational time needed for the simulations but, on the other hand, it limits the amount of features that can be examined in the DAF tank. Bearing in mind the present computational tools, it is not possible to combine detailed physicochemical modeling with 3D geometry models, and this is the reason for the extensive use of 2D models in literature. However, in this study, we had to analyse a portion of the flotation system in 3D to meet certain objectives.

\section{Choice of the turbulence model}

In a next step in building a numerical model of the DAF unit, the choice of a turbulence model needs to be made. The problem of modeling turbulence plays an important role in this work. The reason is that the contact zone is an important mixing zone in the tank and, as such, the flow field in that zone should be well resolved. Here, enough mixing is needed for the air bubbles and solid particles to attach and form aggregates, but at the same time, the mixing should not be too intensive to not break the flocculated aggregates. In DAF in general, there are no external driving mixing mechanisms such as stirrers or baffles. Therefore, turbulence plays a vital part in the removal mechanisms of the flocs (Fawcett 1997) and, consequently, its modeling has to be carefully addressed when formulating a CFD model. Due to the confined geometry in the pneumatic nozzle regions, the flow in the contact zone is treated as a wall-bounded flow. In this work, we have tested only the standard $k-\varepsilon$ model over other turbulence models as it is well established in the wall bounded flows.

\section{GOVERNING EQUATIONS, BOUNDARY CONDITIONS AND FLUID PROPERTIES}

In general, flotation involves three phases and requires independent sets of momentum equations for the liquid, gas, and solid phases. However, for DAF applications in water and wastewater treatment, the mass and volume fractions of particles are very small and, in addition, their size is too small for considerable sedimentation so the solid phase can be treated as a passive scalar. So the problem is to solve the 
two-phase problem for the gas and liquid phase. In most commercial CFD codes, there are three different approaches to deal with the problem: (i) the Lagrangian-Eulerian (Brennen 2005) approach is of stochastic nature and has a great success in simulating, e.g. bubble column behaviour, but is very time-consuming and limited to low gas volume fractions $(<0.1)$; (ii) the two-fluid Eulerian-Eulerian approach is a deterministic one and consists of considering the phases as interpenetrating continua with their own set of momentum equations. The method is still under development, and it is greatly based on empiricism; and (iii) the Mixture model which is also a deterministic one of Eulerian type. This method assumes that all phases behave as a mixture and solves only one set of momentum equations using the concept of drift and slip velocities. All other variables are found from those of the mixture using standard algebraic relations. Though it has some disadvantages, it is much less computationally demanding compared to the other two approaches and hence is used in this project. The governing equations for the Mixture model are as follows:

Continuity $: \frac{\partial \rho_{m}}{\partial t}+\nabla \cdot\left(\rho_{m} \bar{u}_{m}\right)=0$

Momentum:

$$
\begin{aligned}
& \frac{\partial \rho \bar{u}_{m}}{\partial t}+\nabla \cdot\left(\rho_{m} \bar{u}_{m} \bar{u}_{m}\right)=-\nabla p+\nabla\left[\mu_{e f f}\left(\nabla \bar{u}_{m}+\nabla u_{m}^{T}\right)\right]+\rho_{m} g \\
& +F+\nabla \sum_{k=1}^{n} \alpha_{k \rho_{k}} u_{k}^{r} u_{k}^{r}
\end{aligned}
$$

Transport of volume fraction of each secondary phase:

$$
\frac{\partial\left(\alpha_{p} \rho_{p}\right)}{\partial t}+\nabla \cdot\left(\alpha_{p} \rho_{p} \bar{u}_{m}\right)=-\nabla \cdot\left(\alpha_{p} \rho_{p} u_{p}^{r}\right)
$$

where the symbols have their usual meanings. The addition of a third phase (floc/solid particles) in the Mixture model can be assumed as dispersed, carried along the main flow and is solved as a separate phase, incorporating the DPM (discrete phase model) solver. The turbulent dispersion of particles is modelled using a stochastic approach, in which the instantaneous turbulent velocities is calculated from a number of local quantities, viz. turbulent kinetic energy, Eulerian time scale and distance to the wall. The stochastic model used here belongs to the discrete random walk models (Gosman \& Ioannides I983), in which dispersion of particles is understood as a sequence of interactions between a particle and eddies having finite lengths and lifetimes. The characteristic lifetime and the eddy are calculated as:

$\tau_{e}=C_{l} \frac{k}{\varepsilon} ; \quad L_{e}=C_{\mu}^{0.5} \frac{k^{1.5}}{\varepsilon}$

where $k$ and $\varepsilon$ denote the turbulent kinetic energy and dissipation. ' $C_{l}$ ' and ' $C_{\mu}$ ' represent constant and variable of the turbulent model chosen. Here, the interaction between the continuous (gas) and disperse phases (particles) is accounted for. This implies that the particle loading is such that a feedback on the carrier phase is to be expected. On the other hand, the interactions, viz. collisions or coalescence, are not explicitly modelled in the present work as those models would require ample development time. Also, the particle loading of the dispersed phase is limited to $<0.01$ which is a pre-requisite condition of the DPM solver. The details on the flow solvers relate to Mixture and the DPM model can be found from the user's guide manual (Ansys User's Guide, 20I7).

\section{Modelling the flotation}

In the CFD code, the magnitudes of the difference between the bubble and the liquid velocity vectors and between the particle and the liquid velocity vectors are used. The second difference must be equivalent to the settling velocity, but the first one may include an additional relative motion of the bubble due to its inertia. The local gas hold up and turbulent energy dissipation rate needed for the computation of the local flotation frequency are computed by the CFD code. Furthermore, the rise velocity of bubbles and the floc-bubble aggregates is calculated using the following equations (Haarhoff \& Edzwald 2004).

$$
\begin{gathered}
v_{b}=\frac{g\left(\rho_{w}-\rho_{b}\right) d_{b}^{2}}{18 \mu_{w}} \quad v_{f b}=\frac{4 g\left(\rho_{w}-\rho_{f b}\right) d_{f b}^{2}}{3 K \mu_{w}} \\
d_{f b}=\left(d_{f}^{3}+N d_{b}^{3}\right)^{1 / 3} \quad \rho_{f b}=\frac{\rho_{f} d_{f}^{3}+N \rho_{b} d_{b}^{3}}{d_{f}^{3}+N d_{b}^{3}}
\end{gathered}
$$

where $v_{f b}$ is the floc-bubble aggregate rise velocity, $K$ accounts for floc shape and drag force, $d_{f b}$ is the aggregate equivalent diameter produced from $\mathrm{N}$ bubbles of size $d_{b}$ attached to a floc particle of size $d_{p}$ and $\rho_{f b}$ floc-bubble aggregate density. For further flocs-bubble attachment, the readers may refer to the work done by Koh \& Schwarz (2006). Also, the interactions (collisions or coalescences) are also important which may affect the 
flotation-kinetics which is not explicitly modelled in the present study.

\section{Computational domain}

Visualising the flow features in a 3D flotation tank is more realistic especially, the spreading of the supersaturated airflows in the tank width. Since we are more interested in the flow dynamics in the contact and separation zones, the computational domain is restricted to these zones. Also, a high computational cost restricts us for choosing a lower order model (here, $1 / 10^{\text {th }}$ section of the DAF system) for the $3 \mathrm{D}$ analysis. The fluid from the flocculation units is fed through the inlet and the clean water is leaving from the outlet. There are two vertical baffles near the water inlet of the tank to control the fluid flow. The one on the left is fixed vertically, whereas the one on the right is inclined at certain angles. Due to the baffles' arrangement, the liquid which enters the tank is forced to travel first through a down flow section and then through an up flow section. These two sections constitute the reaction zone of the tank. Solid particles are assumed to enter the tank as a homogeneous mixture with the incoming water. The dimensions of the inlet, outlet, contact and separation zones, etc. are described in the Table 1 . To avoid any numerical divergence, the inlet and outlet lengths have been enlarged, which is common to the CFD modelling practice.

\section{Modelling the nozzle (PAN) configuration}

The concept of pneumatically adjustable nozzle (PAN) has been developed which allows the saturation flow to be adjusted automatically to changes in raw water flow and/ or quality (Cromphout \& Vandenbroucke 2000). The orifice is formed by a gap between a solid part of the nozzle body and a rubber diaphragm. By increasing the pneumatic pressure on the dry side of the diaphragm, the orifice opening, and hence the saturation flow, is decreased. To decrease the level of turbulence at the exit of the nozzles and thereby minimizing the risk of floc damage due to excessive shear forces, the nozzles are equipped with an external shroud. A technical drawing of the PAN nozzle is shown in the Figure 1 as below.

The flotation units are equipped with 40 PAN nozzles in two rows (each row contains 20 nozzles) along the width direction and are operated at a varying flow rate $(\sim 200$ to $500 \mathrm{~m}^{3} / \mathrm{h}$ per unit) while keeping the recycle ratio at constant values, resulting in good flotation performance. Considering the domain symmetry in the $1 / 10^{\text {th }}$ section model, only four pneumatic nozzles are considered for the 3D simulation. The supersaturated air is emanating from the adjacent six holes per nozzle (except the central hole). The mass flow rates across each PAN nozzle is calculated, considering the $6 \%$ of the recycle flow rate.

\section{Modelling the degas region}

In the DAF utility, the top surface of the separation zone which is generally filled with the sludge layers, is quite below the top concrete wall and is thus behaves nearly a free surface. To model this, we considered a degas zone (of 1 unit thickness in the height (y) directions from the actual top surface) that extends from the contact to the outlet zones. The degas region appears as a rectangular
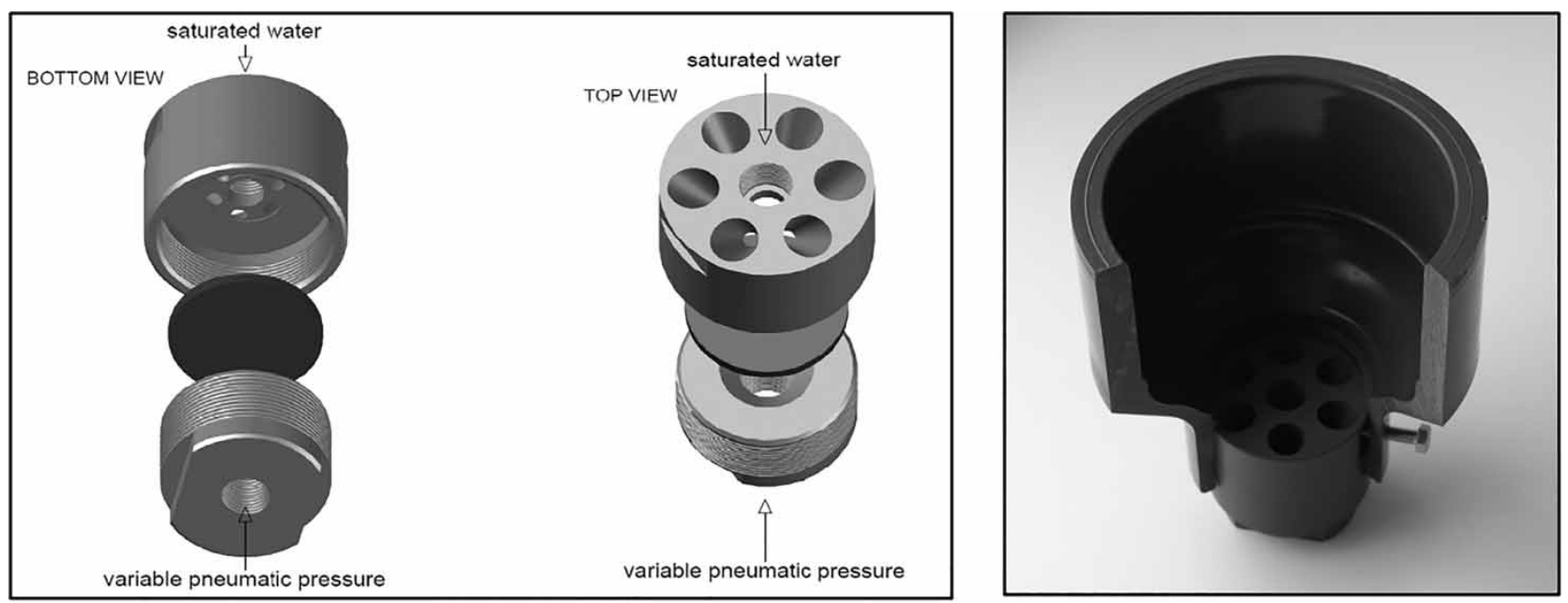

Figure 1 (left) Schematic of the PAN nozzle and (right) the nozzle after installation of the outlet shroud. 
cuboid. The top surface of the degas zone is modelled as a wall with zero shear. An UDF is written and is compiled with the numerical model that acts as a sink for the gas phase and also allows the floc particles floats in this region.

\section{Global grid pattern}

Prior to analyzing the DAF studies in a 3D CFD platform, extensive studies were done in a $2 \mathrm{D}$ geometry (viz., grid independence test, numerical validation against the published literature, baffle elevations, effect of bubble and particle diameters, flow rates, etc. which are not presented in the current manuscript). The 2D optimized mesh was selected as a base case for the 3D domain (viz., horizontal, and perpendicular direction). The number of mesh elements in the other dimension, i.e. $\mathrm{z}$ direction, is necessarily refined to achieve a better convergence. Also, the mesh near the walls was also necessarily refined w.r.t the $\mathrm{y}^{+}$value. Since, the $k-\varepsilon$ turbulence model is used, hence the $\mathrm{y}^{+}$value is respected with the desired limit. The most difficult part is to mesh the present design before switching to the solver settings for the 3D CFD analysis. The usage of PAN nozzles in the $3 \mathrm{D}$ model is another important challenge with respect to design. Therefore, maximum effort has been spent on designing the model and on the meshing. Considering the multiphase nature of the process, the meshes are necessarily refined near the injectors, contact and, in the upper portion, the separation zones to capture the important phenomena. The Auto CAD model is used for the CAD design and the meshing and simulations were performed using the commercial CFD package ANSYS Workbench/ANSYS Fluent 18.0. The optimised mesh for the present $1 / 10^{\text {th }}$ sectional DAF model consists of hexahedral elements and is $\sim 1,265,000$ and the global grid pattern is depicted in the Figure 2.

\section{Boundary conditions}

Towards the boundary condition, the inlet is prescribed as the velocity inlet with $10 \%$ turbulence intensity whereas the outlet is maintained as the pressure outlet with $10 \%$ backflow turbulence intensity. The injectors are maintained as velocity inlets. The remaining walls (except the top which is modelled as a free slip wall) are maintained with no-slip conditions. For the flocs/particles, the inlet and walls of the DAF system, reflect boundary condition are imposed where as the outlet is prescribed with the escape boundary condition. The trap boundary conditions are imposed at the top and bottom surface of the separation zone in order to minimize the flocs $(\%)$ through the outlet towards achieving a better flotation system. The fluids and the flocs properties considered for the analysis are listed in the Table 2. The air volume fraction at the injectors is $1 \%$ which is determined from the degree of oversaturation of the recycle flow rate. The flocs is around $43 \mathrm{ppm}$ of the inlet mass flow rate.

\section{RESULTS AND DISCUSSION}

\section{Model validation}

Experimental validation is an important aspect in a CFD study. A measurement campaign was successfully conducted with the actual flow conditions using the ADV (Sontek). The usages of the ADV allow to collect high-resolution velocity in a rapidly changing environment and are suitable to perform measurements near walls, baffles, etc. The ADV was used to collect 3D velocity data of the liquid phase. The ADV probe (Figure 3) operates by the principle of Doppler shift and records instantaneous velocity components $\left(\mathrm{V}_{\mathrm{x}}, \mathrm{V}_{\mathrm{y}}, \mathrm{V}_{\mathrm{z}}\right)$ at a single point. The probe consists

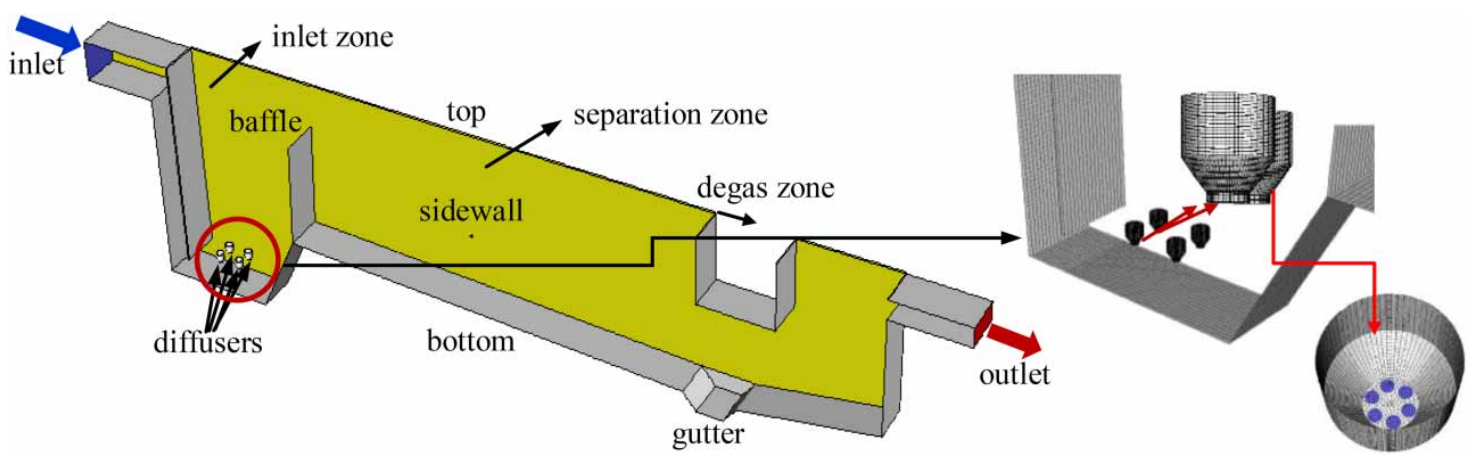

Figure 2 | Side view of the 3D DAF model with global grid patterns in and around the diffusers. 
Table 2 | Fluids and the flocs properties

\begin{tabular}{lllll} 
& Density $\left(\mathbf{k g} / \mathbf{m}^{\mathbf{3}}\right)$ & Viscosity $(\mathbf{K g}-\mathbf{m} / \mathbf{s})$ & Mass flow rate $\left(\mathbf{m}^{\mathbf{3}} / \mathbf{h}\right)$ & Recycle flow rate $(\%)$ \\
\hline Water & 998 & 0.001 & 500 & 6 \\
Air & 1.225 & $1.89 \mathrm{E}-5$ & & 20 \\
Flocs/particle & 1,100 & - & & 100 \\
\hline
\end{tabular}

of one transmitter and three receivers. The sampling volume is located $10 \mathrm{~cm}$ from the tip of the transmitter, and is a cylinder with a diameter of $0.6 \mathrm{~cm}$ and a height of $0.7 \mathrm{~cm}$. The transmitter emits single frequency acoustic waves $(10 \mathrm{MHz})$ to the sampling volume where particles scatter the acoustic wave. The three receivers 'listen' for the change in frequency of the returned waves. The frequency of the Doppler shift between the transmitted and received acoustic pulses allows for calculation of the instantaneous velocities in the sampling volume. The velocity range of the ADV device is $\pm 0.001-4.5 \mathrm{~m} / \mathrm{s}$, and its accuracy is $\pm 1 \%$ of the measured velocity. All the measurements were carried out with the same configuration.

Since the instrument is very sensitive on the design perspective (probe locations), any minor error/mishandles of the instrument would cause a serious damage to the instrument. Hence, design of additional protecting covers and supporting structures were made on the ADV surface for usage in the DAF system. The details on the supporting structures are described in a separate sheet (Appendix 1, Figures 13 and 14). In order to check the function of the ADV, initial attempts were made by conducting trial measurements in the lab and in a large pool with clean water medium and the results were found to be satisfactory (not included in the report) and gave a better confidence.
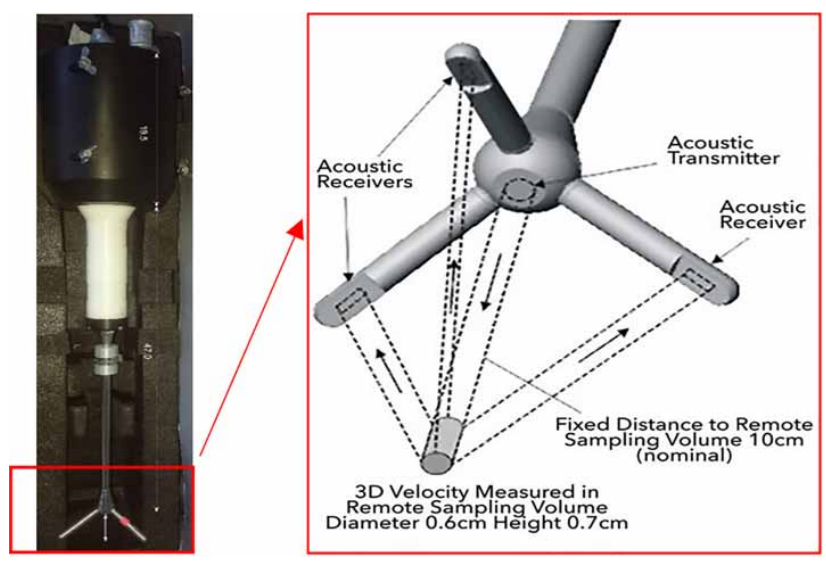

Figure 3 ADV device with the probes.

\section{Measurement campaigns}

Setting-up the measurement campaign inside the treatment plant was not straightforward due to the complex design, especially the lamellas configurations. As a priori, the measurement locations, height and depth of the probe locations, flow rates, etc. were pre-determined. Also, measurements were restricted only near to the side walls due to the absence of any substantial wall support structures for the ADV to avoid any further damage to the instrument. The set-up of the ADV device in the DAF system is depicted in Figure 4.

The co-ordinates $\mathrm{x}, \mathrm{y}, \mathrm{z}$ denote the flow in the axial, vertical and tangential directions. The device was used in the downlooking position and submerged $47 \mathrm{~cm}$ under the water surface in the DAF. The five measurement locations were chosen along the length of the DAF, located at (i) $2.1 \mathrm{~m}$, (ii) $3.23 \mathrm{~m}$ (iii), $4.05 \mathrm{~m}$, (iv) $5.42 \mathrm{~m}$ and (v) $6 \mathrm{~m}$ from the inlet of the basin. Details are listed in Table 3. For each location, $\pm 5,000$ measurements were recorded.

\section{Error analysis}

The ADV probe is prone to different kinds of errors in the velocity output signal and hence raw ADV velocity data

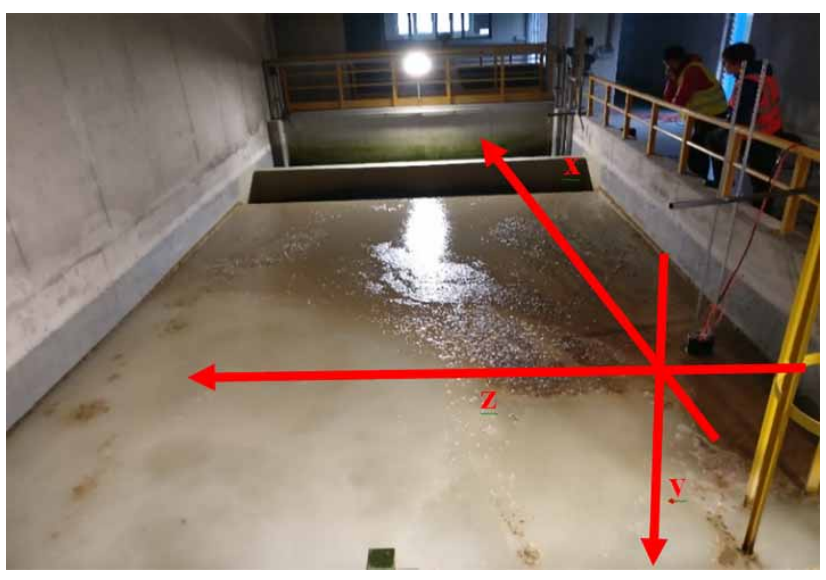

Figure 4 Schematic of the measurement campagain with the directions at the DAF utility. 
Table 3 | Detailed measurements conducted inside the treatment plant

\begin{tabular}{|c|c|c|c|c|c|}
\hline \multirow[b]{2}{*}{ SI. No. } & \multirow{2}{*}{$\begin{array}{l}\text { Flow rate } \\
\left(\mathrm{m}^{3} / \mathbf{h}\right)\end{array}$} & \multirow{2}{*}{$\begin{array}{l}\text { Recycle flow } \\
\text { rate (\%) }\end{array}$} & \multicolumn{3}{|c|}{ Coordinates } \\
\hline & & & $x(m)$ & $y(m)$ & $z(m)$ \\
\hline 1 & 500 & 6 & 2.10 & -0.47 & 0.425 \\
\hline 2 & 500 & 6 & 3.23 & -0.47 & 0.425 \\
\hline 3 & 500 & 6 & 4.05 & -0.47 & 0.425 \\
\hline 4 & 500 & 6 & 5.42 & -0.47 & 0.425 \\
\hline 5 & 500 & 6 & 6.00 & -0.47 & 0.425 \\
\hline
\end{tabular}

typically contain noise. Potential sources of errors could be the appearance of spikes due to lack of tracer particles in the sampling volume or Doppler noise (while noise inherent to acoustic instruments). Therefore, the ADV velocity timeseries were filtered based on the signal-to-noise (SNR) ratio and correlation (recorded by the instruments). The lower limits for correlation and SNR of $70 \%$ and $5 \mathrm{~dB}$ were set. Figure 5 shows a samples of raw (a) and filtered (b) 3D velocity time series at the first measurement point (2.1 $\mathrm{m}$ from the DAF inlet).
The recorded mean correlation values are found to be $43.67 \%$ \& $41.0 \%$, respectively, indicating the noise disturbances.

The velocity data was analysed and the result is presented in Figure 6 . Since the tangential component magnitude is negligible, focus is on the axial and normal components. It is observed that the magnitude and direction of the velocity at the locations of $2.1 \mathrm{~m}, 4.05 \mathrm{~m}, 5.23 \mathrm{~m}$ and $6.0 \mathrm{~m}$ are comparable. The deviation of velocities at some locations may be due to the limitation of the ADV probe location near to the side wall of the DAF utility, lamellas configurations (which are not included in the current design) as well as the 3D nature of the flow features. Overall, the experimental findings are found to be highly satisfactory.

\section{D Flow features with reference design (3D.0)}

In the flotation system, three distinct flow regimes exist, viz., inlet zone, contact and the separation zone. The flow features in the separation zone are an important aspect in the flotation system. The velocity distribution in the tank is depicted in Figure 7(a).
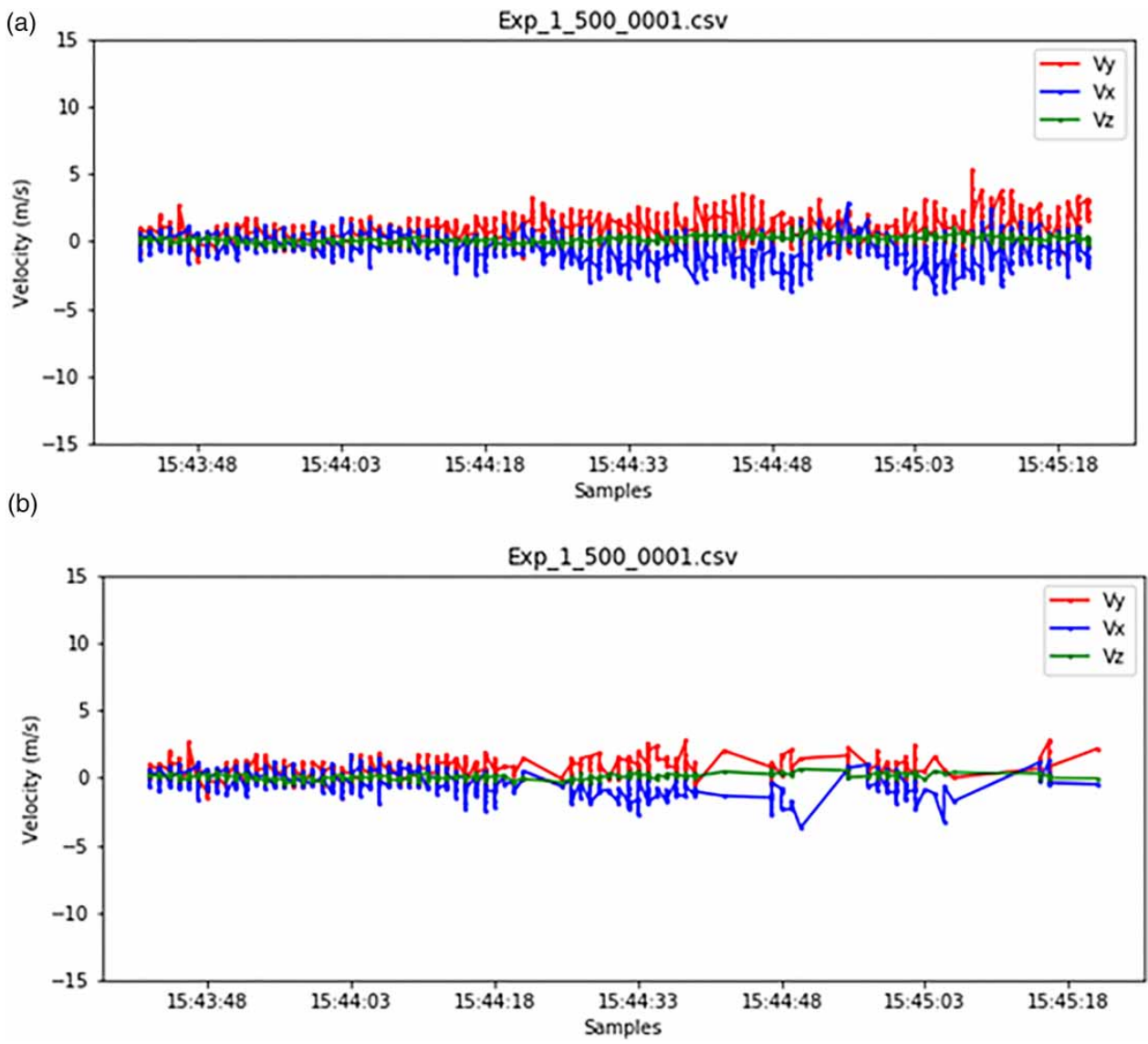

Figure 5 | ADV time-series obtained in the DAF, $2.10 \mathrm{~m}$ from the inlet (a) raw data-set; (b) clean data after filtration. 


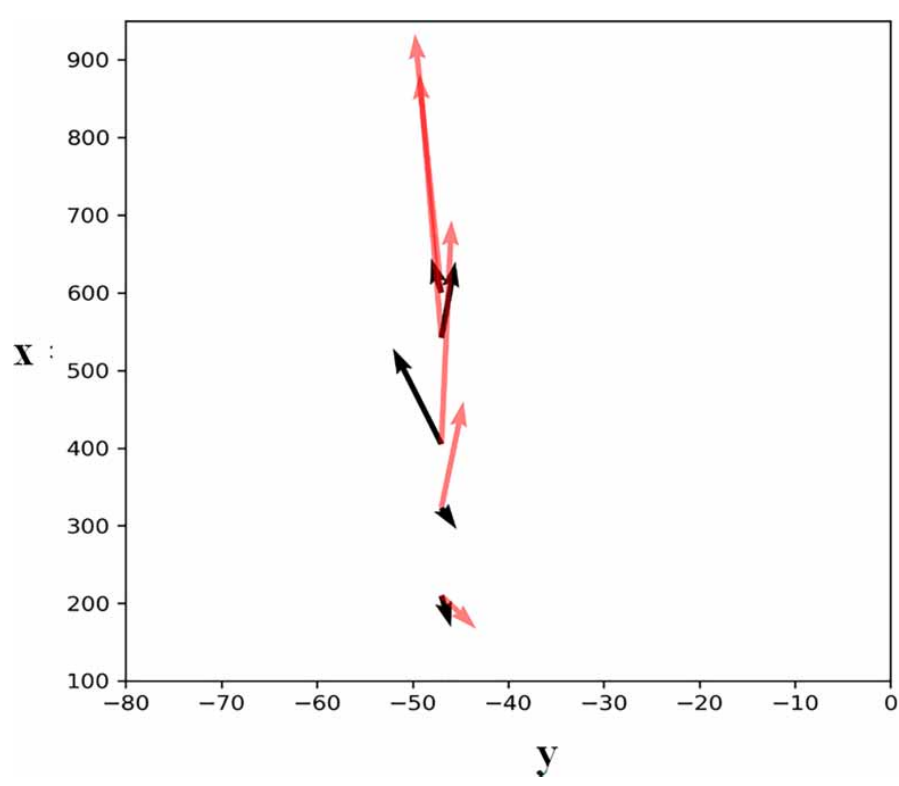

Figure 6 | Velocity vectors from the ADV data (mean velocities) against the CFD results.

The fluid (water) emanating from the inlet zone follows a downward movement and enters the contact zone through a narrow passage. It travels along the baffle plate against the gravity and finally enters the separation zone. Higher velocity regimes appear near the bottom of the contact zone. Two large recirculation zones exist in the system: in the contact zone and in the separation zone and followed by a small recirculation near the outlet. The contour of air void fractions is depicted in Figure 7(b). It is seen that the air fraction is maximal near the diffusers and it spreads throughout the contact zone and finally reaches the top free surface. Subsequently, it confines within the degas zone. A very small fraction of air bubbles $(<1 \%)$ follows the fluid paths along the separation zone (due to the dominance of

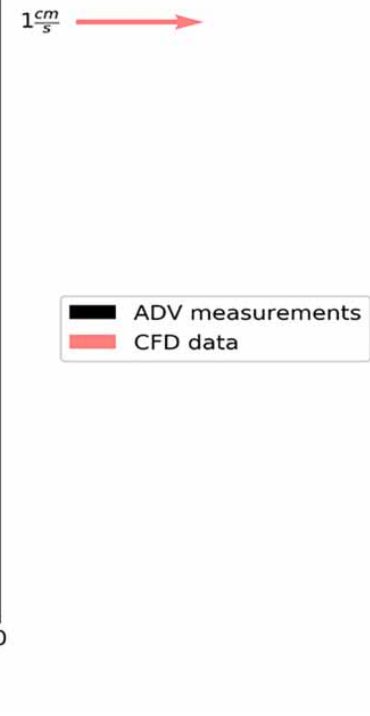

tangential fluid velocity over the bubble buoyancy) and finally reaches the outlet. The particle concentration in terms of particle residence time (s) is plotted in the Figure 8.

The colour contour shows the flocs flow path with a uniform time frame in the DAF system. At the beginning (0-125 s), the flocs follow the water motion, following a downward movement and reaching the bottom of the contact zone. In the subsequent intervals (125-375 s), they partly remain in the contact zone where bubble-flocs attachment occurs and partly escape to the free surface forming the froth layer. Some flocs follow the flow path in the separation zone and get trapped at the bottom of the separation zone. In the interval up to $500 \mathrm{~s}$, the flocs, which are not able to get trapped either in the froth layer or the bottom of the
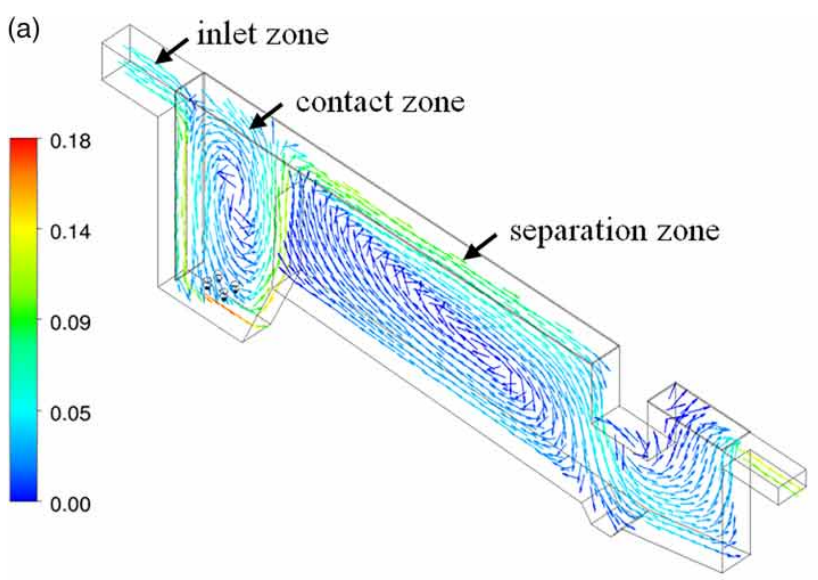

Figure 7 | (a) velocity (m/s) and (b) air void fraction inside the domain. (b)

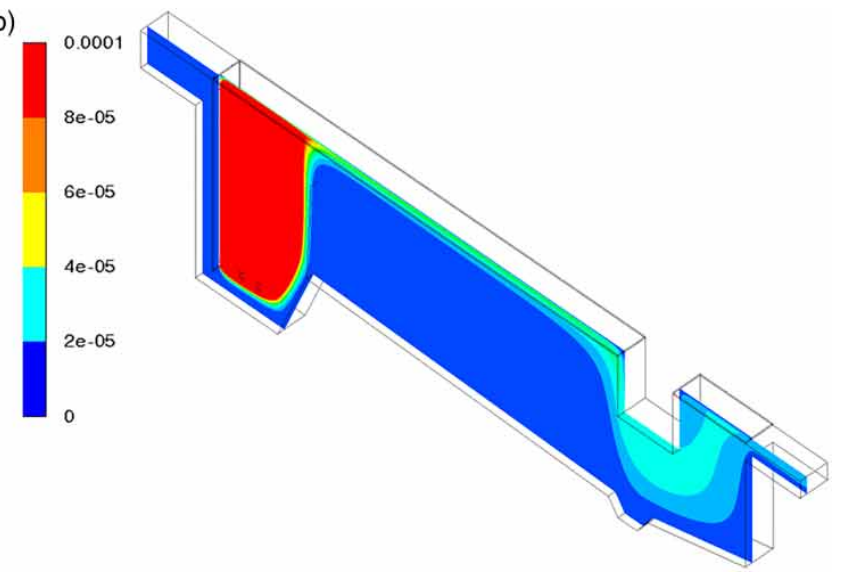




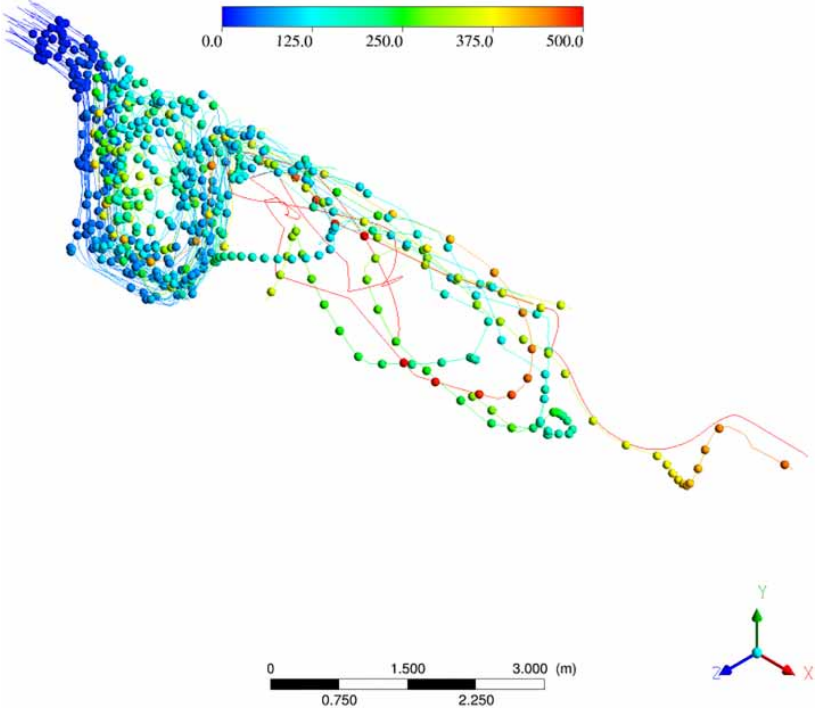

Figure 8 | Particle tracks colored by particle residence time (s) for the reference design.

separation zone, finally escape through the outlet. The trapping probability is found to be $64 \%$ (i.e. escape is $36 \%$ ).

\section{Design modifications}

Since the previous reference design (3D.0) is prone to higher particle escape, which is adverse for the DAF performance, it needs to be minimised. Towards this, a new design has been proposed. Here, the fluid extraction is brought about by means of a perforated pipe and the outlet section of the DAF is discarded. A uniform discharge over the entire length of the pipe is assumed which gives a geometrical set-up as illustrated in Figure 9(a).

The dimensions of the inlet zone, contact zone and air diffusers remain identical as the reference design. Since the design of the separation zone is modified with the

(a)

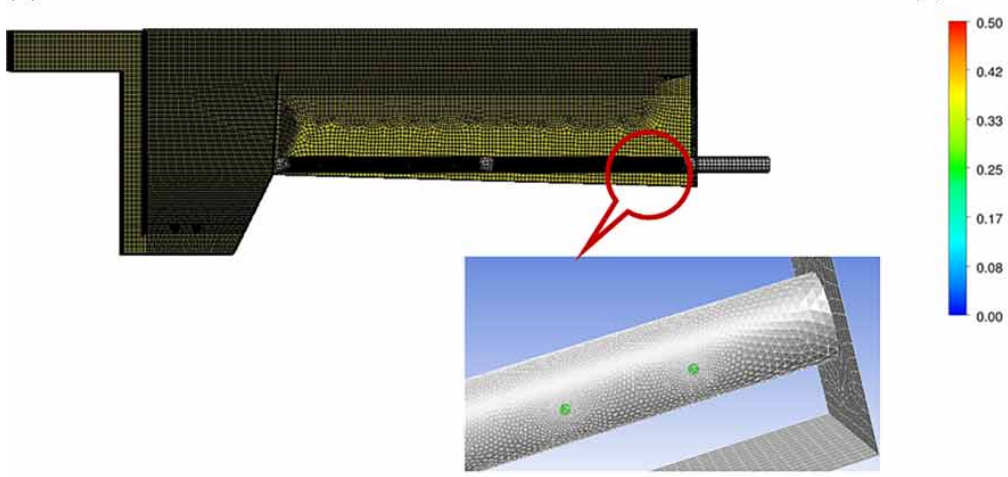

Figure 9 | (a) Design modifications with a perforated tube and (b) velocity distributions. perforated pipe and with a flat right wall, its axial length decreased from the reference design. The perforated pipe is modelled as a thin circular tube with symmetric uniform holes distributed sidewise along the pipe wall. The diameter of the pipe and holes are $0.2 \mathrm{~m}$ and $0.025 \mathrm{~m}$, respectively. The pipe covers the entire span of the separation length and is placed just above the bottom of the separation zone. Here, the inlet loads, bubble and particle diameters and its concentrations remain same as the reference scenario (3D.0). All major boundary conditions remain the same. Additional interior boundary conditions are specified on the perforated hole faces to allow the fluids to pass. In order to avoid numerical diffusion, the outlet is enlarged to a few centimetres from the original design. The different case studies under the proposed design modifications are presented in the Table 4.

\section{Flow features through a fully perforated tube (3D.1)}

From the velocity distributions, it is seen that a large recirculation appears in the separation zone (see the red arrow mark in Figure 9(b)). The center of the recirculation appears to be more or less at the center of the separation zone. At the bottom of the separation zone, an accelerated velocity region exists at the separation bottom (marked by the black arrow line) which extends throughout the separation zone. Moreover, a velocity gradient prevails along the entire tube length, mainly due to the presence of very small hole openings. The information on the air void fraction and the particles residence time are presented in the Figure 10(a) and 10(b), respectively.

The void fractions contour shows a similar behavior as the reference scenario (3D.0). The higher downward velocity near the outlet contributes the air bubble escape but

(b)

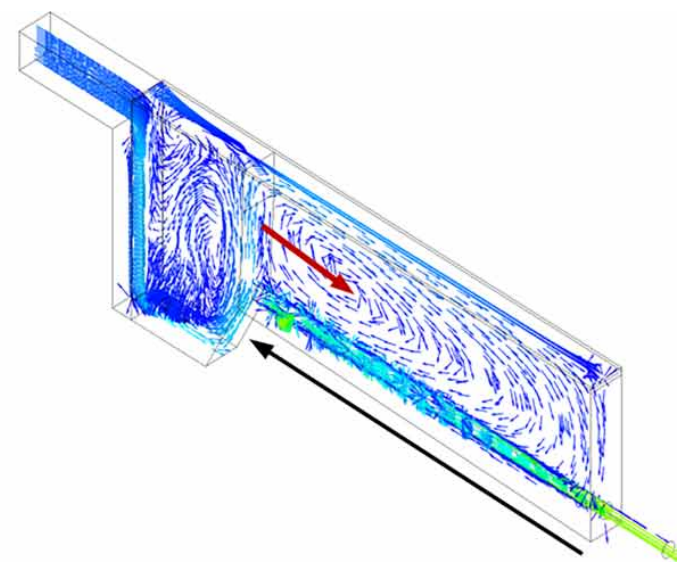


Table 4 | Case studies under the proposed 3D design modifications

\begin{tabular}{llllll}
$\begin{array}{l}\text { Different } \\
\text { scenario }\end{array}$ & Pipe Perforation (\%) & $\begin{array}{l}\text { Tube } \\
\text { dia. }(\mathbf{m})\end{array}$ & $\begin{array}{l}\text { Hole dia. } \\
(\mathbf{m})\end{array}$ & $\begin{array}{l}\text { Holes } \\
\mathbf{g a p}(\mathbf{m})\end{array}$ & $\begin{array}{l}\text { Inlet } \\
\mathbf{l o a d s} \\
\left(\mathbf{m}^{\mathbf{3}} \mathbf{h}\right)\end{array}$ \\
\hline 3D.1 & 100 & 0.2 & 0.025 & 0.3 & 500 \\
3D.2 & $\begin{array}{c}50 \text { (perforation near } \\
\text { the outlet) }\end{array}$ & & & & 500 \\
3D.3 & $\begin{array}{c}50 \text { (perforation near } \\
\text { the baffle wall) }\end{array}$ & & & \\
$\quad$ & & & & \\
\hline
\end{tabular}

the percentage is very low. The flocs trapped at the free surface is $\sim 79 \%$ which performs much better compared to the reference design without the perforated tube.

\section{Flow features through a partially perforated tube} (3D.2, 3D.3)

Given the fact that the solid particles are mostly following the downward flow in the separation zone and, hence, most likely escape through the tube outlet due to high inward velocities at the small openings across the perforated tube. A limited number of scenarios could be tested in order to limit the particle escape through the outlet at a lower value. For this, the efficiency of a partly closed perforated tube over the fully perforated one has been investigated. Two different case studies were analysed, i.e. (i) the perforation covers $50 \%$ of the separation zone and is near to the outlet (3D.2) and (ii) 50\% perforation in the region closest to the baffle (3D.3). The flow features (velocity) and the particle concentrations (DPM) are depicted in Figures 11 and 12, respectively. Air void fractions distribution for these cases resembles the reference case (not shown).

The flow pattern behaves similarly to the earlier scenario (3D.1), but the vortex core is now shifted far from the baffle wall as well as at a lower level from the free surface (Figure 11(a)). The shifting of the vortex core enforces a higher velocity magnitude through the perforated tube. This seems logical as the same volume of liquid per unit

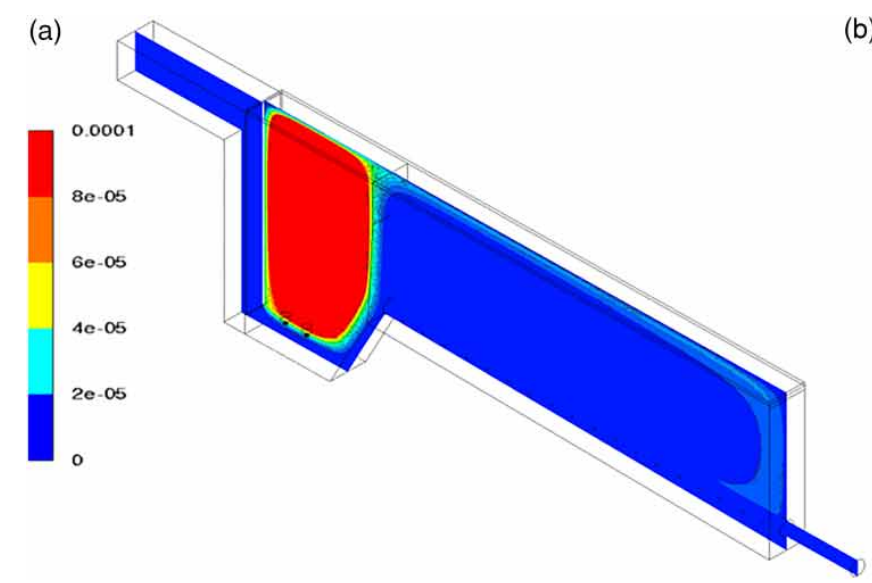

(b)

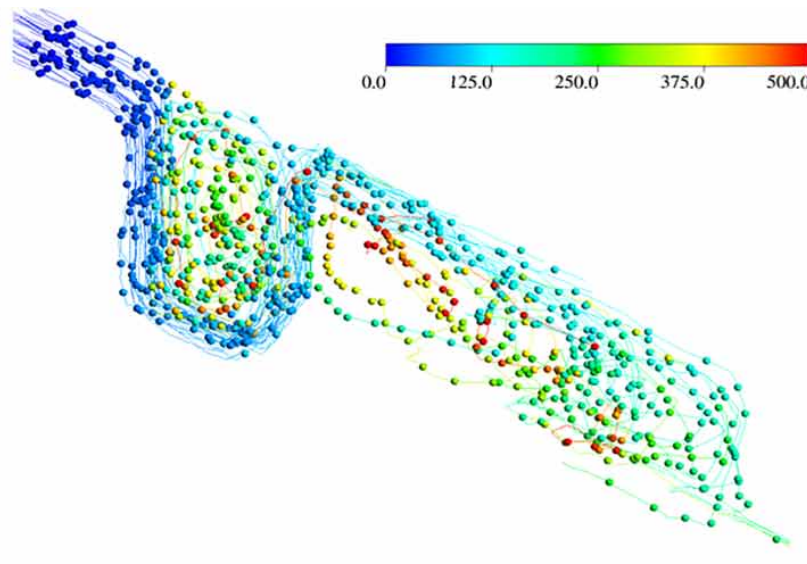

Figure 10 | (a) Air void fraction and (b) particles residence time for the perforated tube design.

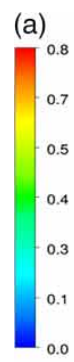

(b)

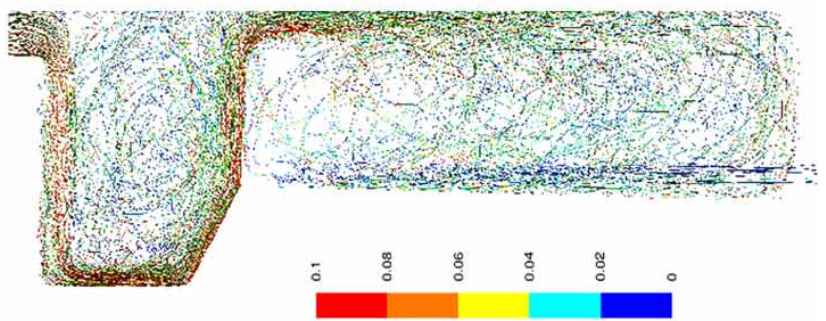

Figure 11 | (a) Velocity vectors (m/s) and (b) DPM concentrations (kg/s) for the scenario 3D.2. 
(a)

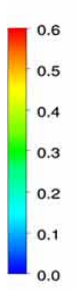

(b)

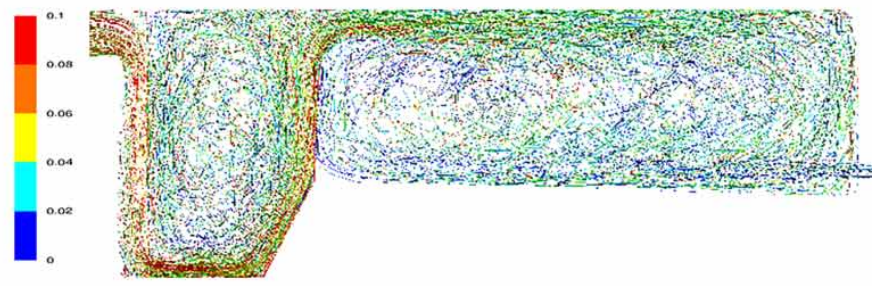

Figure 12 | (a) Velocity vectors (m/s) and (b) DPM concentrations (kg/s) for the scenario 3D.3.
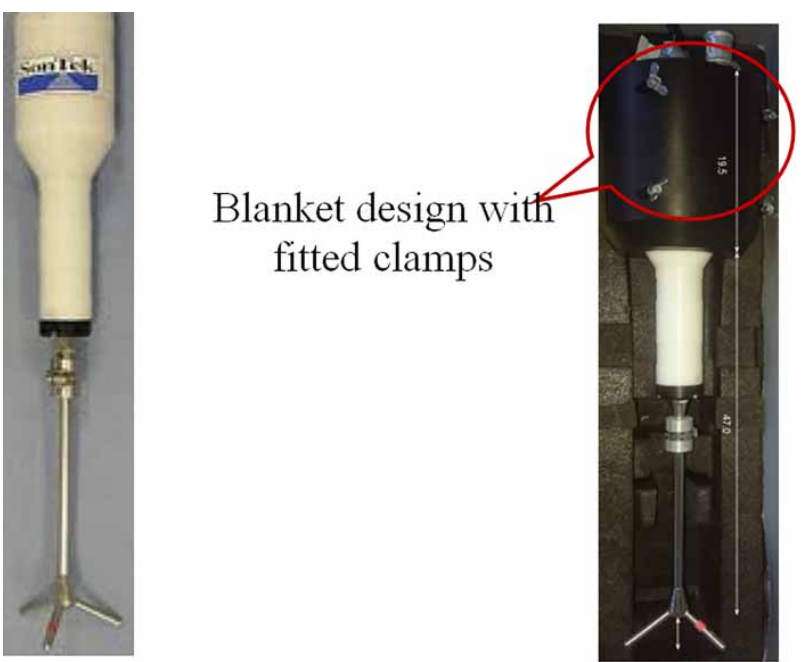

Figure 13 | Additional designs made in the UGent Design Workshop (left) without the blanket and, (right) ADV with the blanket cover. of time needs to be extracted through half the surface area compared to the previous case. Since the available surface area is lower in the perforated tube for the flow to be fully developed, it is less likely for solid particles to escape through the outlet. The trapped percentage is $\sim 86 \%$ (Figure 11(b)) which is much higher than the former scenarios.

In the case of partial perforation near to the baffle wall (3D.3), the vortex core is now shifted towards the baffle wall as marked by the thick red arrow line. The flow enters inside the perforated tube with high velocity, but attains a uniform magnitude across the other end of the separation zone, i.e. up to the outlet (Figure 12(a)). The velocity is of lower magnitude as compared to scenario 3D.2. Below the perforated tube, there is complete absence of any accelerated velocity regimes indicating the flow is quite uniform. Here, large surface area/volume available (a)

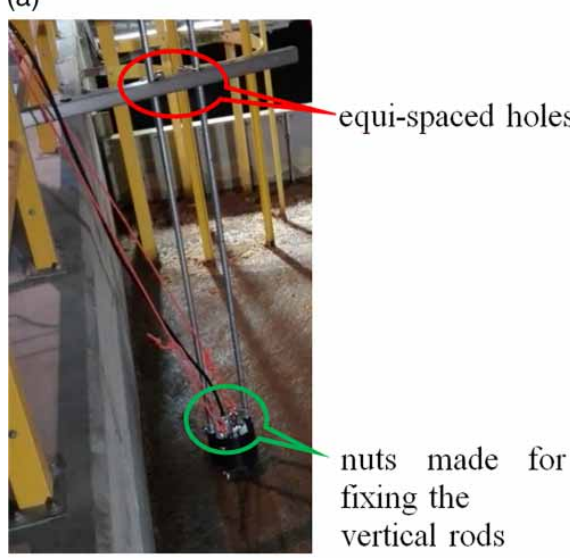

(b)

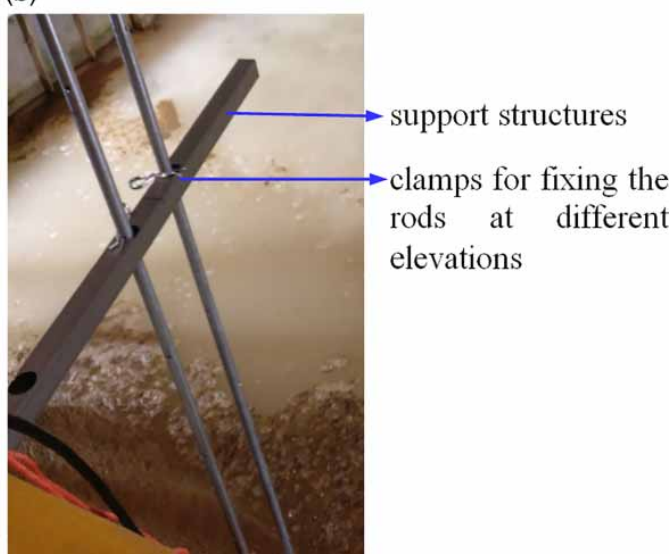

Figure 14 (a) Support structures made on the top and, (b) along the rods for holding/fixing the ADV at different elevations. 
for the flow to be fully developed (marked by the arrow mark in the yellow velocity regimes inside the tube), the tube acts more or less like a vacuum cleaner and hence more particles escape through the outlet (Figure 12(b)). The trapping probability is $\sim 82 \%$.

\section{RECOMMENDATIONS}

Comparing the above design scenarios of using the perforated tube for improved DAF design, the partial perforation with opening at the outlet seems to be promising (in terms of flocs trapped) for future DAF designs. It is noteworthy that the flotation kinetics might be prone to some uncertainty. The small scale phenomena such as bubble-particle collisions, effect of particle relaxation time on the bubble-particle coalescences etc., were not considered in detail. These effects may affect the flocs trapping at the free surface. However, the observed trends should still hold. Also, further measurement campaigns with data analysis will be a suitable choice of another domain of research.

\section{CONCLUSION}

3D CFD simulations were carried out on a $1 / 10^{\text {th }}$ DAF section model to get an insight of the flow features inside the treatment plant. The flow features from the numerical analysis gives a better confidence, but particle escape through the system outlet still prevails, hampering the system performance. To improve the performance, design modifications are tested by using a perforated tube to extract the water. It is found that the usage of a partially perforated tube open to the system outlet performs better and hence, may be a suitable option for future DAF designs. The discussion is further reinforced through validating the numerical model against the experimental findings for stratified flow conditions and the comparison is found to be highly satisfactory.

\section{ACKNOWLEDGEMENT}

The authors are thankful to Ir. S. Balemans, Ir. David F. del Pozo and Dr. W. Naessens for their contribution during the measurement campaigns.

\section{SUPPLEMENTARY MATERIAL}

The Supplementary Material for this paper is available online at https://dx.doi.org/10.2166/wst.2020.028.

\section{REFERENCES}

Amato, T. \& Wicks, J. 2007 The practical application of computational fluid dynamics to dissolved air flotation plant operation, design and development. In: Proc., 5th Int. Conf. on Flotation in Water and Wastewater Systems. Seoul National University, Seoul, 105-112.

ANSYS User's Guide 2017 Ansys, Inc. Lebanon, USA.

Bondelind, M., Sasic, S. \& Peterson, T. J. R. 20Io Setting up a numerical model of a DAF tank: turbulence, geometry, and bubble size. J. Environ. Eng. 136 (12), 1424-1434.

Brennen, C. E. 2005 Fundamentals of Multiphase Flows. Cambridge University Press, Pasadena, CA, USA.

Cromphout, J. \& Vandenbroucke, S. 2000 Design of an automatically adjustable flotation nozzle. In: Proc. of the 4th Int. Conference on Flotation in Water and Waste Water Treatment, Helsinki, Finland.

Edzwald, J. K. 1995 Principle and applications of DAF. Water Sci. Technol. 31 (3-4), 1-23.

Edzwald, J. K., Malley, J. P. \& Yu, C. I99o A conceptual model for dissolved air flotation in water treatment. Water Supply $\mathbf{8}$, 141-150.

Emmanouil, V., Skaperdas, E. P., Karapantsios, T. D. \& Matis, K. A. 2007 Two-phase simulations of an off-nominally operating dissolved-air flotation tank. Int. J. Environ. Pollut. 30 (2), 213-230

Fawcett, N. S. J. 1997 The hydraulics of flotation tanks: Computational modelling. In: Proc. Dissolved Air Flotation Conf. The Chartered Institution of Water and Environmental Management, London, UK, 51-71.

Gosman, A. D. \& Ioannides, E. I983 Aspects of computer simulation of liquid-fuelled combustors. J. Energy 7 (6), 482-490.

Guichao, W., Linhan, G., Subhasish, M., Geoffrey, M. E., Joshi, J. B. \& Songying, C. 2018 A review of CFD modelling studies on the flotation process. Minerals Eng. 127, 153-177.

Haarhoff, J. \& Edzwald, J. K. 2004 Dissolved air flotation modelling: insights and shortcomings. J. Water Supply: Res. Technol. AQUA 53 (3), 127-150.

Hague, J., Ta, C. T. \& Biggs, M. J. 20or Small scale model for CFD validation in DAF application. Water Sci. Technol. 43 (8), 167-173.

Koh, P. T. L. \& Schwarz, M. P. 2006 CFD modelling of bubbleparticle attachments in flotation cells. Minerals Eng. 19 (6-8), 619-626.

Kwon, S. B., Park, N. S., Lee, S. J., Ahn, H. W. \& Wang, C. K. 2006 Examining the effect of length/width ratio on the hydrodynamic behaviour in a DAF system using CFD and ADV techniques. Water Sci. Technol. 53 (7), 141-149. 
Lundh, M. 2000 Flow Structures in Dissolved air Flotation. Licentiate thesis, Lund University, Sweden.

Ta, C. T. \& Brignal, W. J. 1997 Application of single phase CFD techniques to dissolved air flotation tank studies. In: Conference on Dissolved Air Flotation, London, 472-488.
Verdickt, L., Ferroukhi, N., Moreau, J., Besson, A., Guiraud, P. \& Cromphout, J. 2016 Characterization of a pneumatically adjustable saturation nozzle for dissolved air flotation. In: 7th Int. Conference on Flotation in Water \& Wastewater Systems, Toulouse, France.

First received 28 October 2019; accepted in revised form 20 January 2020. Available online 28 January 2020 